\title{
Structural characterization of gel-grown neodymium copper oxalate single crystals
}

\author{
K S RAJU ${ }^{+}, \mathrm{K}$ N KRISHNA*, JAYAKUMARI ISSAC** and \\ M A ITTYACHEN** \\ Department of Crystallography and Biophysics, University of Madras, Guindy Campus, \\ Madras 600025, India \\ * Materials Research Centre, Indian Institute of Science, Bangalore 560012, India \\ ** School of Pure and Applied Physics, Mahatma Gandhi University, Kottayam 686031, \\ India \\ MS received 10 May 1994; revised 14 July 1994
}

\begin{abstract}
Sparingly soluble neodymium copper oxalate (NCO) single crystals were grown by gel method, by the diffusion of a mixture of neodymium nitrate and cupric nitrate into the set gel containing oxalic acid. Tabular crystal, revealing well-defined dissolution figures has been recorded. X-ray diffraction studies of the powdered sample reveal that NCO is crystalline. Infrared absorption spectrum confirmed the formation of oxalato complex with water of crystallization, while energy dispersive $X$-ray analysis established the presence of neodymium dominant over copper in the sample. X-ray photoelectron spectroscopic studies established the presence of $\mathrm{Nd}$ and $\mathrm{Cu}$ in oxide states besides $\left(\mathrm{C}_{2} \mathrm{O}_{4}\right)^{2-}$ oxalate group. The intensities of $\mathrm{Nd}\left(3 d_{5 / 2}\right)$ and $\mathrm{Cu}\left(2 p_{3 / 2}\right)$ peaks measured in terms of maximum photoelectron count rates also revealed the presence of $\mathrm{Nd}$ in predominance. The inductively coupled plasma analysis supports the EDAX and XPS data by the estimation of neodymium percentage by weight to that of copper present in the NCO sample. On the basis of these findings, an empirical structure for NCO has been proposed. The implications are discussed.
\end{abstract}

Keywords. Neodymium copper oxalate; gel growth; characterization; XRD; IR; EDAX; XPS.

\section{Introduction}

The growth of sparingly soluble inorganic crystals employing gel technique (Henisch 1970 ) is well established and extensive work has been reported in the literature on the morphologies (Raju 1983a, b, c, 1986), nature of dislocations (Raju 1985), as well as on the mechanical behaviour (Raju et al 1984) in the case of gel-grown calcium sulphate dihydrate single crystals. It is well known that the rare-earth compounds exhibit high-temperature superconductivity and oxalate crystals show good ionic conductivity (George Verghese et al 1990), hence it is interesting to study the characteristics of rare-earth mixed oxalates. Caillaud et al (1988) have shown that superconducting compounds can be obtained by the controlled precipitation of oxalates followed by calcination. Further, researchers have proved that oxides like lanthanum copper oxide exhibit superconductivity up to $40 \mathrm{~K}$ (Beille et al 1987).

We report here the work carried out on the gel-grown mixed oxalate crystals of copper and neodymium (neodymium copper oxalate, $\mathrm{NCO}$ ) and their characterization employing X-ray diffraction (XRD), IR, energy dispersive X-ray analysis (EDAX), $X$-ray photoelectron spectroscopy (XPS) and inductively coupled plasma (ICP)

\footnotetext{
${ }^{+}$Address for correspondence
} 
studies, which throw light on the kinetics of chemical reactions, the qualitative and quantitative analyses of heavy elements present in the mixed oxalates, besides the presence of their chemical states.

These results help in speculating on the empirical structure of NCO sample.

\section{Experimental observations and results}

\subsection{Growth of NCO in gels}

Acidified silica gel of specific gravity $1.03 \mathrm{~g} \mathrm{~cm}^{-3}$ was used as the reacting medium. A mixed solution of neodymium nitrate and cupric nitrate with nitric acid was used as the outer reactant above the gel at room temperature. The outer reactant diffused into the set gel containing oxalic acid. The following reaction takes place, producing bluish pink colloidal precipitate of mixed oxalates of neodymium and copper $\left(\mathrm{Nd}_{2} \mathrm{Cu}_{3}\left(\mathrm{C}_{2} \mathrm{O}_{4}\right)_{6} \cdot n \mathrm{H}_{2} \mathrm{O}\right)$ and nitric acid:

$$
\left.\begin{array}{c}
2 \mathrm{Nd}\left(\mathrm{NO}_{3}\right)_{3} \\
+ \\
3 \mathrm{Cu}\left(\mathrm{NO}_{3}\right)_{2}
\end{array}\right]+6 \mathrm{H}_{2}\left(\mathrm{C}_{2} \mathrm{O}_{4}\right) \longrightarrow \mathrm{Nd}_{2} \mathrm{Cu}_{3}\left(\mathrm{C}_{2} \mathrm{O}_{4}\right)_{6} \cdot n \mathrm{H}_{2} \mathrm{O}+12 \mathrm{HNO}_{3}
$$

The nitric acid present in the gel medium may induce nucleation from the precipitate resulting in crystallization. A large number of well-defined bluish pink transparent microcrystals were observed just below the interface, while isolated crystals of relatively bigger sizes appeared at greater depths in the gel. The optimum conditions for the growth of good single crystals were found to be $\mathrm{pH} 5$ of the gel and 0.5 molarity of each of the nitrates in $1: 1$ by volume in the outer reactant.

The proposed empirical structure of neodymium copper oxalate is given in figure 1 .

Figure 2 shows an SEM of the basal plane of an NCO crystal (of size $5 \times 3 \times 0.5 \mathrm{~mm}^{3}$ ) grown at greater depth in the gel. The basal plane can be seen to have relatively curved edges and corners. Careful examination of microstructures on the basal plane reveals crystallographically oriented dissolution figures (figure 3) suggesting thereby the single-crystalline nature of the NCO sample.

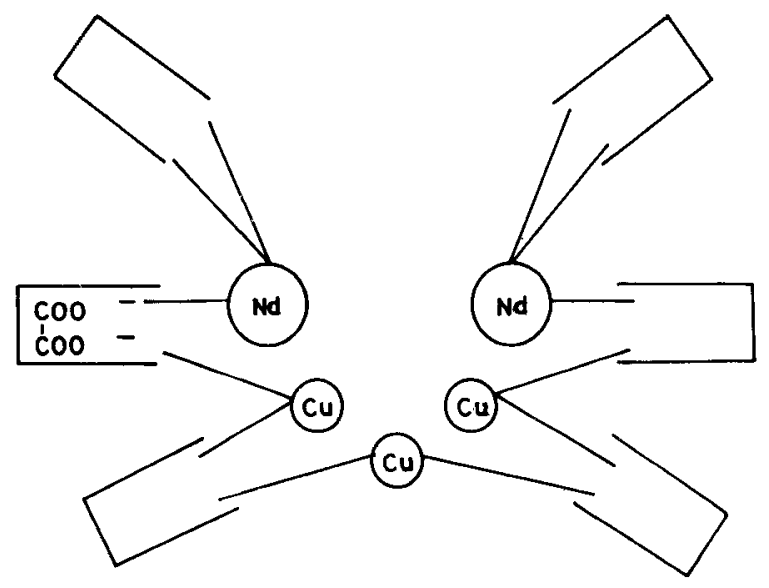

Figure 1. Proposed empirical structure of NCO. 


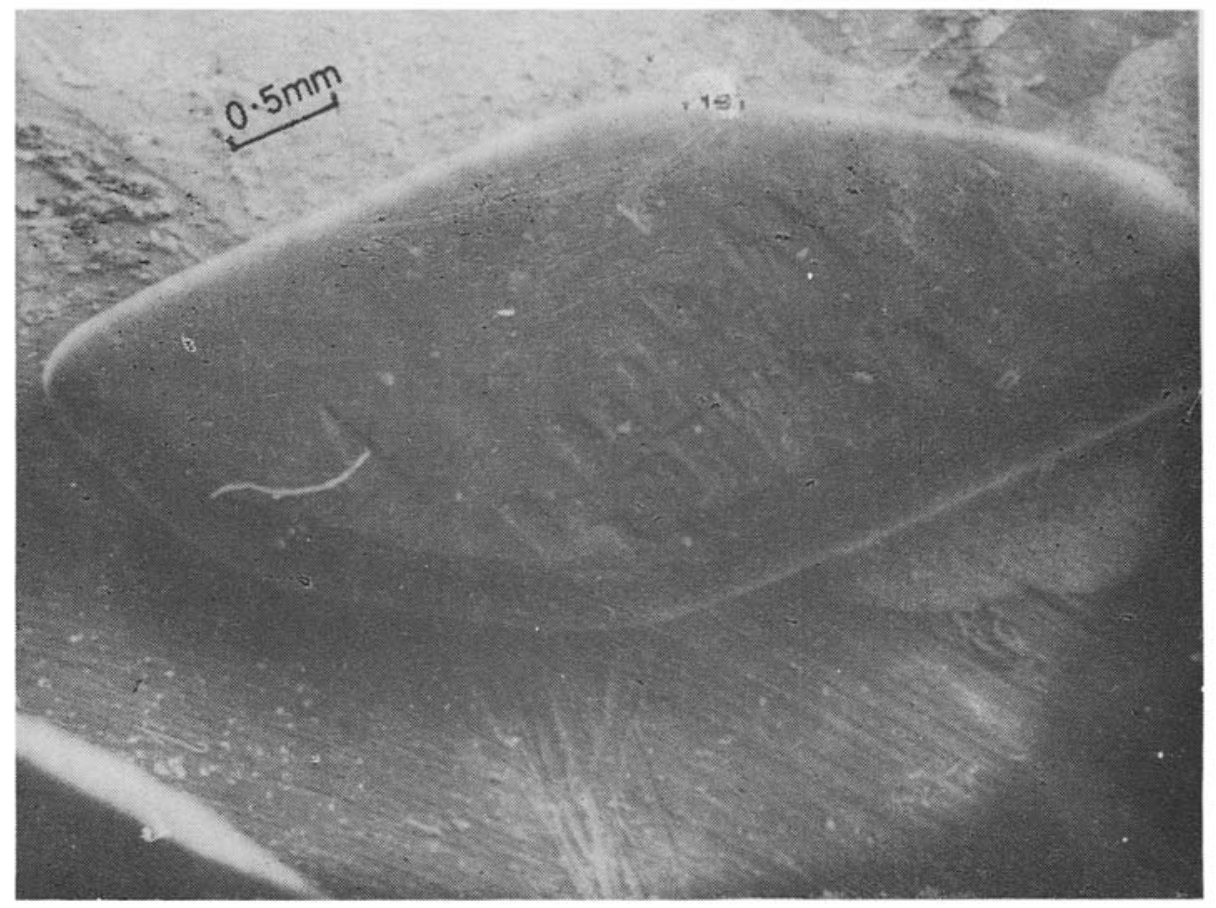

Figure 2. SEM picture of gel grown NCO.

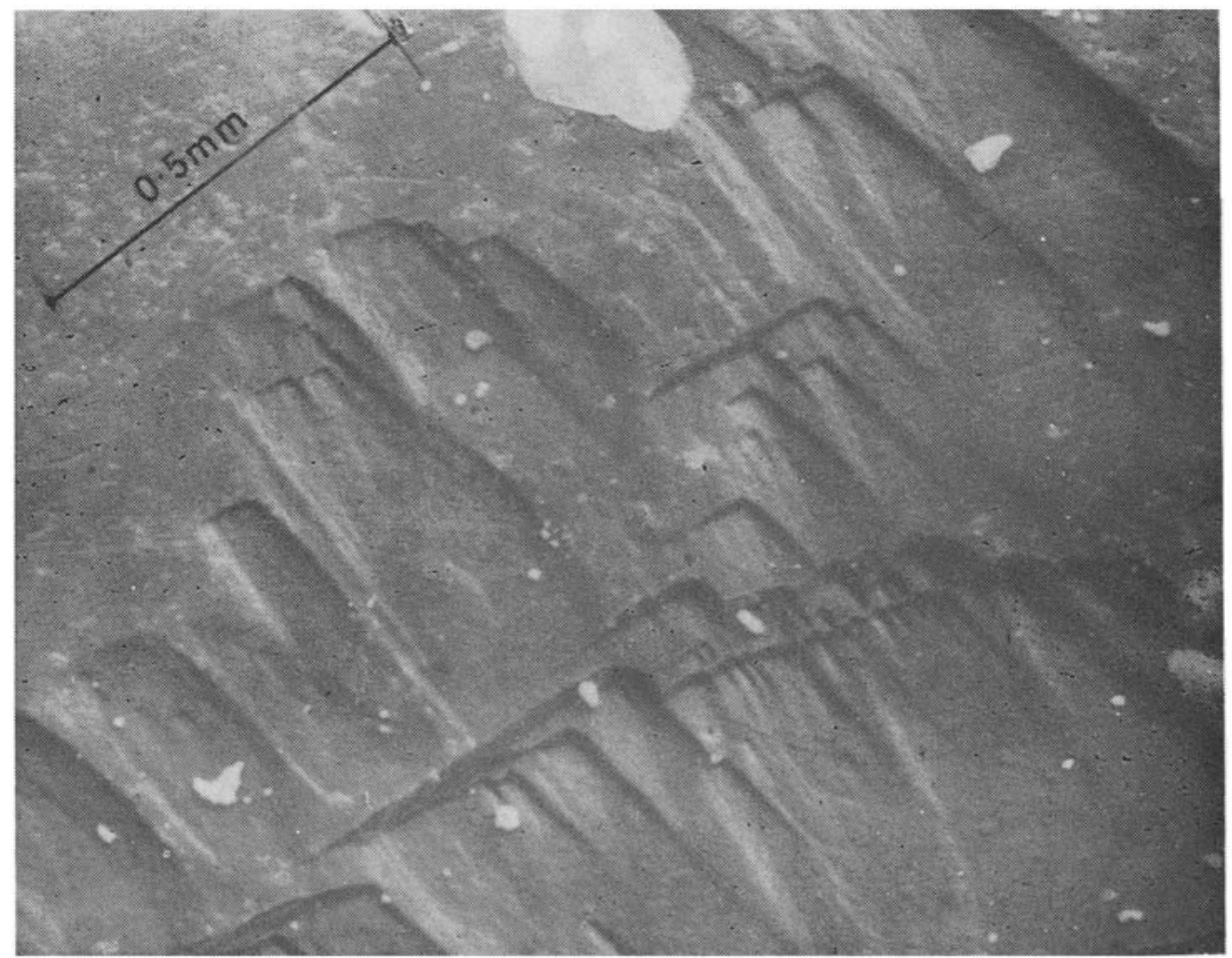

Figure 3. SEM picture showing dissolution figures on basal plane of NCO. 


\subsection{Characterization of $\mathrm{NCO}$}

2.2a XRD studies: In order to confirm the crystallinity, the powder X-ray diffraction pattern of NCO sample (figure 4) was taken in a Philips XRD PW 1050/70 with $\mathrm{CuK}_{\alpha}$ radiation $(\lambda=1.5418 \AA)$. The revelation of well-defined Bragg peaks at specific $(2 \theta)$ angles suggests that $\mathrm{NCO}$ is ordered, meaning crystalline nature of the sample. It is worth mentioning here that the $d$ values of the Bragg peaks in the XRD pattern of powdered NCO (figure 4) match quite well with the $d$ values of the Bragg peaks relating to the XRD pattern recorded for powdered neodymium oxalate dodecahydrate.

2.2b IR absorption studies: The IR absorption spectrum of NCO sample (figure 5) in $\mathrm{KBr}$ matrix was recorded in a Perkin Elmer model 530.

The sample has water of crystallization as evidenced by the broad peak at $3000-3800 \mathrm{~cm}^{-1}$ (relating to antisymmetric and symmetric $\mathrm{OH}$ stretchings) and at $1630 \mathrm{~cm}^{-1}$ (relating to $\mathrm{HOH}$ bending).

That the NCO sample has oxalate $\left(\mathrm{C}_{2} \mathrm{O}_{4}\right)^{2-}$ ions is supported by the revelation of well-pronounced peaks at $1720 \mathrm{~cm}^{-1}\left[\gamma_{a}(\mathrm{C}=\mathrm{O})\right], 815 \mathrm{~cm}^{-1}[\delta(\mathrm{O}-\mathrm{C}=\mathrm{O})+\gamma(\mathrm{MO})]$, $610 \mathrm{~cm}^{-1}, 500 \mathrm{~cm}^{-1}$ [ring deformation $\left.+\delta(\mathrm{O}-\mathrm{C}=\mathrm{O})\right], 390 \mathrm{~cm}^{-1}[\delta(\mathrm{O}-\mathrm{C}=\mathrm{O})+\gamma(\mathrm{CC})]$ (perhaps crystal water) and $335 \mathrm{~cm}^{-1}(\pi)$.

2.2c Energy dispersive $X$-ray analysis $(E D A X)$ : In order to establish the elemental incorporation (like $\mathrm{Nd}$ and $\mathrm{Cu}$ ) in the NCO crystals, EDAX was done. The integrated counts of X-ray photoelectrons relating to the elemental peaks taken for a definite time interval have been helpful in getting the approximate quantitative content of their respective elements reported in the present investigation.

The tiny crystals of NCO were mounted on to an aluminium stub, the surfaces of which were coated with a thin layer of gold to make them electrically conducting. The surfaces were examined in the EDAX analyser No. 711, an accessory to the Philips SEM model 501. The EDAX picture of NCO so recorded is shown in figure 6.

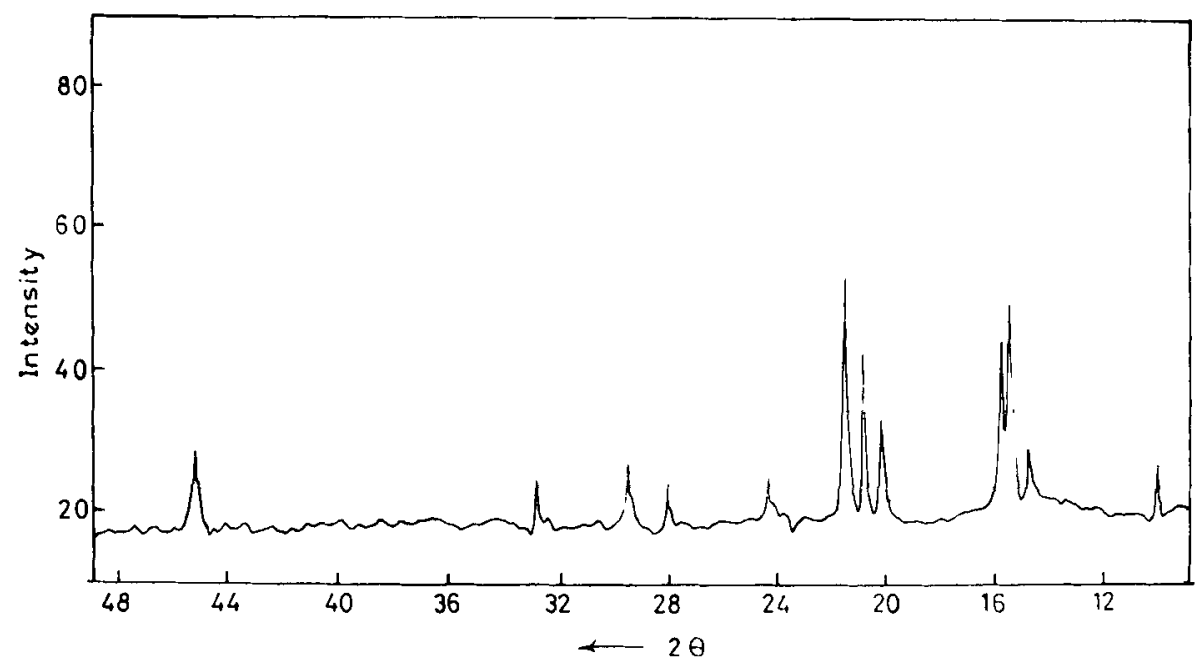

Figure 4. Powder XRD pattern of NCO. 


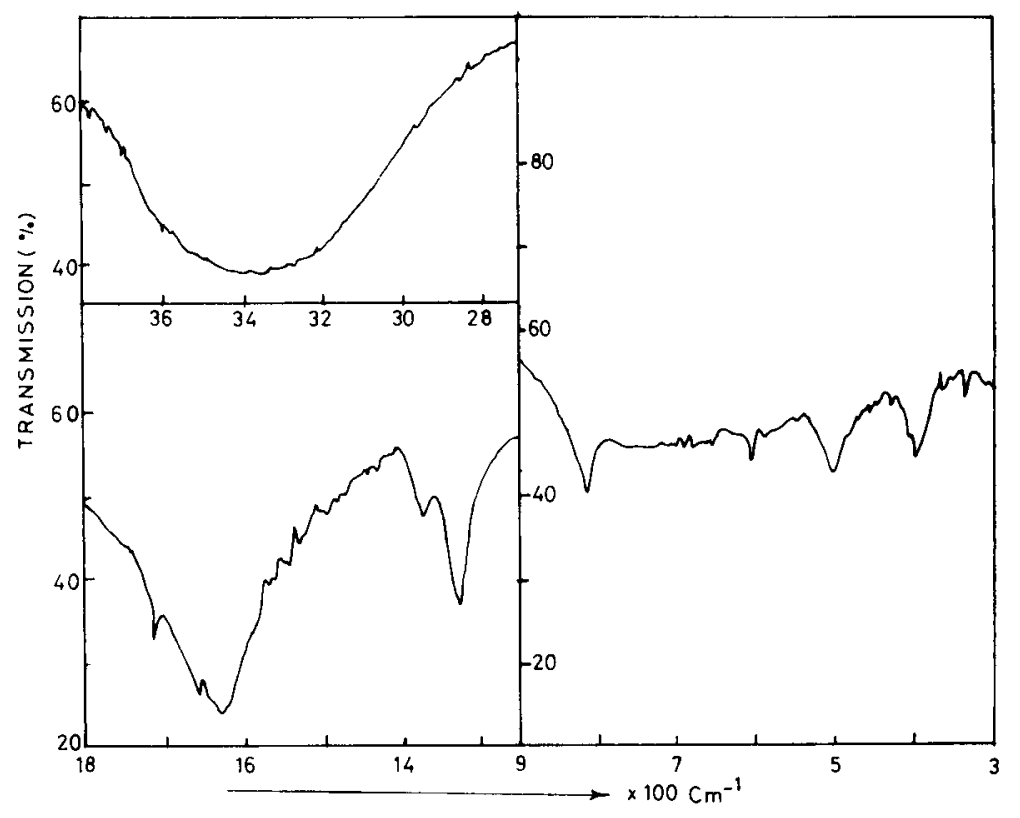

Figure 5. IR absorption spectrum of NCO.

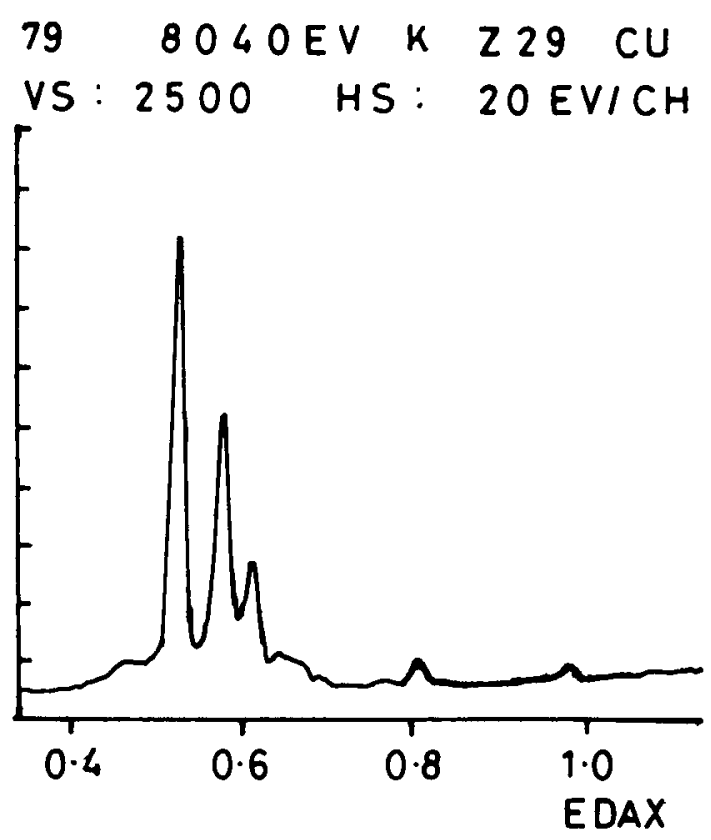

Figure 6. EDAX pattern of NCO.

The three dominant peaks positioned at $5 \cdot 23,5.72$ and $6.088 \mathrm{keV}$ (in the left hand side of figure 6) correspond quite well to the $\mathrm{L}_{\alpha}, \mathrm{L}_{\beta}$ and $\mathrm{L}_{\gamma}$ energies of neodymium, while a small hump at $8.04 \mathrm{keV}$ corresponds to $\mathrm{K}_{\alpha}$ line of copper (reported in the EDAX international chart), thereby establishing the presence of heavy elements (like 
$\mathrm{Nd}$ and $\mathrm{Cu}$ ) in NCO sample. A comparison of heights/areas relating to $\mathrm{L}_{\alpha}$ peak of $\mathrm{Nd}$ and $\mathrm{K}_{\alpha}$ of copper gives a strong clue that neodymium is dominant over copper in the sample.

The integrated count of X-ray photoelectrons relating to neodymium $\left(\mathrm{L}_{\alpha}\right)$ collected for $150 \mathrm{sec}$ was found to be 60,094 while for copper $\left(\mathrm{K}_{\alpha}\right)$ the integrated count was 6144.

A very small hump for copper $K_{\alpha}$ at $8.04 \mathrm{keV}$ compared to neodymium $\mathrm{L}_{\alpha}$ at $5.23 \mathrm{keV}$ observed in figure 6 is supported by the smaller integrated count of X-ray photoelectrons for copper $\mathrm{K}_{\alpha}$ relative to neodymium $\mathrm{L}_{\alpha}$.

2.2d XPS studies: In order to further establish the constituents incorporated in NCO sample besides their chemical states, XPS studies are undertaken.

For the sample preparation, $\mathrm{NCO}$ is nicely powdered and a pellet of $1 \mathrm{~cm}$ diameter and $1 \mathrm{~mm}$ thickness was formed using a hydraulic press by applying a pressure of $1 \cdot 1$ tons per $\mathrm{cm}^{2}$. XPS spectra were taken in VG scientific ESCALAB MK II, by irradiating the sample with monochromatic soft X-rays $(\mathrm{Mg} \mathrm{K}$; photon energy $1253.6 \mathrm{eV})$. The constant analyser energy was $50 \mathrm{eV}$ and the resolution of the system was about $0.7 \mathrm{eV}$.

Figure 7 shows the XPS peak relating to $\mathrm{Nd} 3 d_{5 / 2}$ with the binding energy centred at $980 \mathrm{eV}$, with the maximum count rate (MCR) of photoelectrons 8958 , while figure 8 relates to the XPS peak of $\mathrm{Cu} 2 p_{3 / 2}$ having binding energy around 933.6 eV (MCR 215).

It is well known that the count rates of photoelectrons relating to an XPS peak,
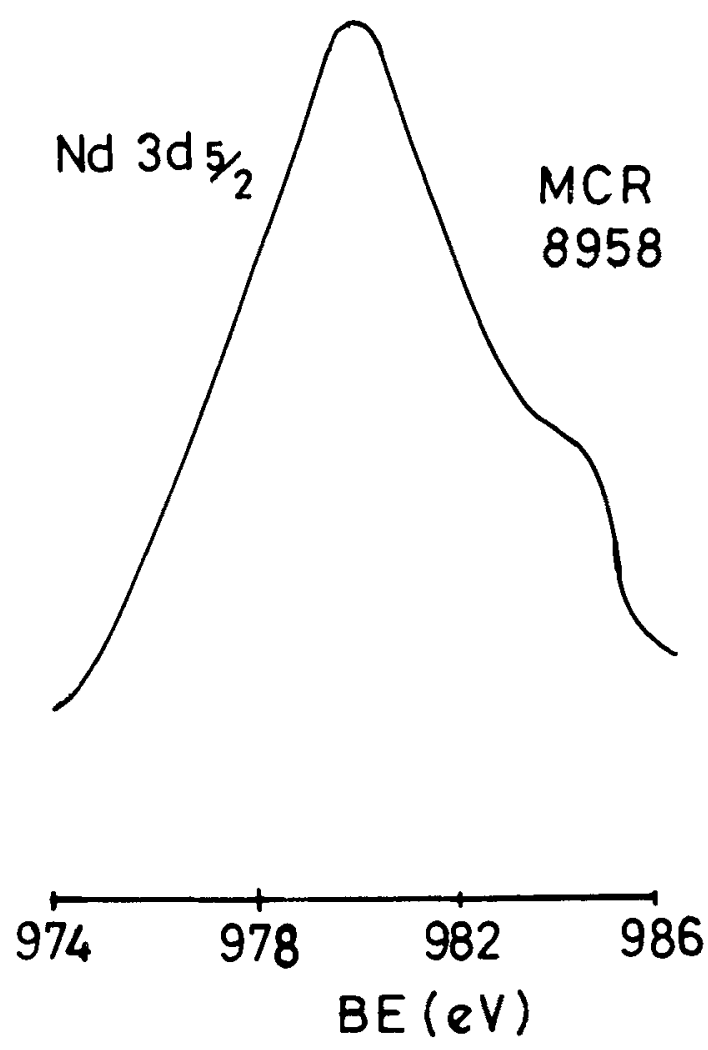

Figure 7. XPS peak of $\mathrm{Nd}\left(3 d_{5 / 2}\right)$ of $\mathrm{NCO}$. 
meaning thereby intensity, is a measure of the quantity of the concerned element present in the material (Riggs and Parker 1975). The XPS observation regarding the MCRs of $\mathrm{Nd}$ and $\mathrm{Cu}$ supports the EDAX observation that $\mathrm{Nd}$ is more dominant than copper in the NCO sample.

The binding energy values relating to $\mathrm{Nd}\left(3 d_{5 / 2}\right)$ and $\mathrm{Cu}\left(3 p_{3 / 2}\right)$ in figures 7 and 8 agree quite well with the literature values (Wagner et al 1978) corresponding to their oxide states $\left(\mathrm{Nd}_{2} \mathrm{O}_{3}\right.$ and $\mathrm{CuO}$ in the present case).

Figure 9 is the XPS region of carbon $1 s$ of NCO sample in which it is clear that there are two peaks at binding energy values 287 and $290 \mathrm{eV}$. The first peak at $287 \mathrm{eV}$ is attributed to the adventitious hydrocarbon being contributed from the laboratory environment or from the glove box, while the latter peak at the higher binding energy $(290 \mathrm{eV})$ relates to the oxalate $\left(\mathrm{C}_{2} \mathrm{O}_{4}\right)^{2-}$ group. This shift towards the higher binding energy corresponding to the oxalate group with respect to the first peak $(287 \mathrm{eV})$ is due to the higher electronegativity of $\left(\mathrm{C}_{2} \mathrm{O}_{4}\right)^{2-}$.

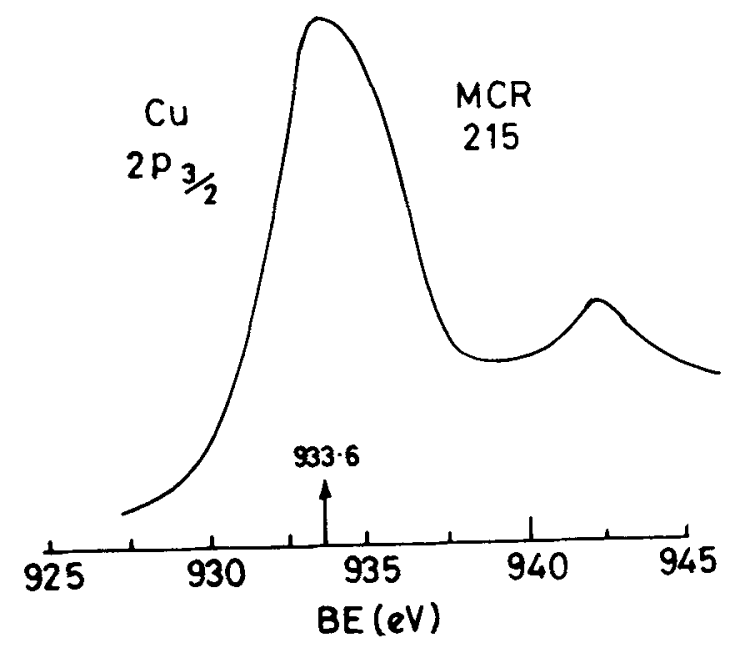

Figure 8. XPS peak of $\mathrm{Cu}\left(2 p_{3 / 2}\right)$ of NCO.

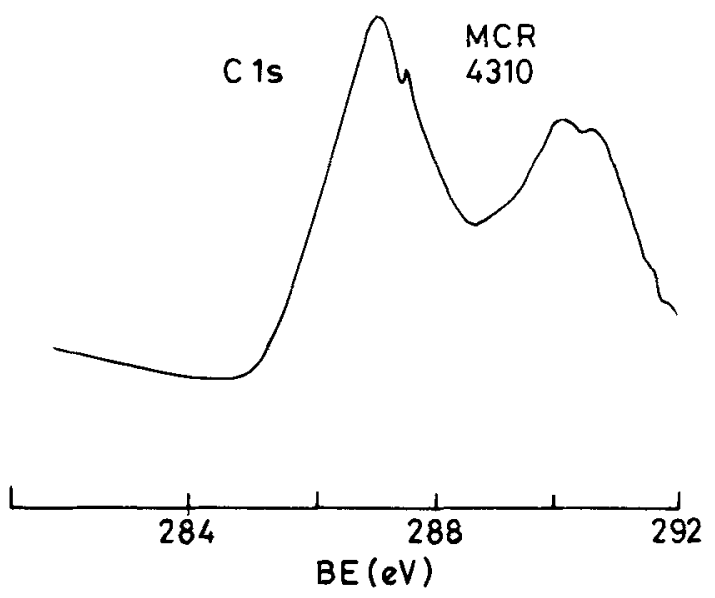

Figure 9. XPS peak of $\mathrm{C}$ is of NCO. 
Thus XPS, though a method for surface analysis, is a powerful technique in identifying not only the constituents present in the sample but also their respective chemical states.

2.2e Inductively coupled plasma (ICP) analysis: The atomic emission spectrum of $\mathrm{NCO}$ by inductively coupled argon plasma was studied. Fifty to $55 \mathrm{mg}$ of NCO was dissolved in $\mathrm{HCl}$ and the solution made up to $25 \mathrm{ml}$. The quantitative analysis of neodymium and copper was carried out with the calibration standards of $\mathrm{Nd}_{2} \mathrm{O}_{3}$ and $\mathrm{CuSO}_{4} \cdot 5 \mathrm{H}_{2} \mathrm{O}$ in the range $0,10,20,50$ and $100 \mathrm{ppm}$. The concentrations of neodymium and copper (in ppm) were found to be 1170 and $6 \mathrm{ppm}$ respectively. These computations are in very good agreement with the findings of EDAX and XPS confirming the dominance of neodymium over copper in NCO sample.

\section{Discussion}

The observation that NCO grown by gel is crystalline in nature is obviously evident by the revelation of tabular crystal at the greater depths in the gel, having geometrical edges and corners. On careful examination of the basal plane of the crystal, a large density of crystallographically oriented dissolution figures was observed. After the cessation of growth, during harvesting of the crystals, the acidic resultant $\left(\mathrm{HNO}_{3}\right)$ in the gel might have sparingly dissolved the sharp edges and corners thereby rounding them (figure 2) and also the basal plane producing crystallographically oriented dissolution figures (figure 3). The crystallinity of the NCO sample was further ascertained by powder XRD (figure 4) which revealed well-defined Bragg peaks whose $d$ values match well with the $d$ values of Bragg peaks relating to powdered neodymium oxalate, which is reported as crystalline (Weast and Lide 1990). The IR absorption spectrum of NCO (figure 5) establishes the presence of oxalato complexes in the sample besides its crystallinity as evidenced by the broad peak $\left(3000-3800 \mathrm{~cm}^{-1}\right)$ relating to the water of crystallization, agreeing quite well with the reported values in the literature (Nakamoto 1978). A small hump at $8.04 \mathrm{keV}\left(\mathrm{K}_{\alpha}\right.$ peak of copper) in EDAX of NCO sample (figure 6) relative to the predominant peak $\mathrm{L}_{\alpha}$ of $\mathrm{Nd}$ is well in agreement with the integrated count of photoelectrons of $\mathrm{L}_{\alpha}$ of neodymium as 60,094 compared to 6144 for $\mathrm{K}_{x}$ of copper, both collected for $150 \mathrm{sec}$. The XPS results support well that $\mathrm{Nd}$ and $\mathrm{Cu}$ are in their oxide states (figures 7 and 8) getting linked to $\mathrm{O}^{-}$of $\left(\mathrm{C}_{2} \mathrm{O}_{4}\right)^{2-}$, the latter being evidenced by the XPS of $\mathrm{C} 1 \mathrm{~s}$ (figure 9). These results are in excellent agreement with the proposed empirical formula for NCO (figure 1). The MCRs of photoelectrons relating to $3 d_{5 / 2}$ peak of neodymium (8958) and $3 p_{32}$ peak of copper (215) reestablish the EDAX and ICP results that $\mathrm{Nd}$ is dominant over copper in NCO sample.

The dominance of $\mathrm{Nd}$ over copper is explained as follows.

The rare earth neodymium is a hard acid and oxalate is a hard base and hence has stronger aftinity for neodymium oxalate formation. Copper belongs to soft acid and in presence of neodymium has a very weak role in the formation of copper oxalate. This may be the reason for the dominance of neodymium over copper in the NCO sample (Huheey 1983). 


\section{Conclusion}

It is concluded that the gel-grown NCO sample reported in the present work (i) is crystalline in nature as evidenced by the well-defined edges and corners (though curved later) as well as by the revelation of crystallographically oriented dissolution figures (figures 2 and 3), (ii) is ordered and crystalline (XRD), (iii) accounts for the presence of oxalato complex by the revelation of its characteristic peaks and of lattice water by the broad peak at $\left(3000-3800 \mathrm{~cm}^{-1}\right)$ (IR), (iv) has $\mathrm{Nd}$ and $\mathrm{Cu}$ established qualitatively and quantitatively (EDAX), (v) has $\mathrm{Nd}$ and $\mathrm{Cu}$ in their oxide states (XPS) getting linked to $\mathrm{O}^{-}$of oxalate groups (figure 1), and (vi) has Nd in abundance relative to $\mathrm{Cu}$ as evidenced by the integrated counts of their X-ray photoelectrons (in EDAX and XPS) and by their quantitative presence estimated by ICP analysis.

\section{Acknowledgements}

KSR thanks the Chairman, Materials Research Centre, Indian Institute of Science, Bangalore, for XRD and IR, Prof. V S Raghunathan, Head, Metallurgy Division, IGCAR, Kalpakkam, for EDAX and RSIC, Indian Institute of Technology, Madras, for XPS and ICP facilities.

\section{References}

Beille J, Cabanel R, Chaillout C, Chevallier B, Demazeau G, Deslandes F, Etourneau J, Lejay P, Michel C, Provost J, Raveau B, Sulpice A, Tholence J L and Tournier R 1987 C. R. Acad. Sci. Ser. 23041097 Caillaud F, Baumard J-F and Smith A 1988 Mater. Res. Bull. 231273

George V. Ittyachen M A and Jayakumari Issac 1990 Cryst. Res. Technol. 25 k-153

Henisch H K 1970 Crystal growth in gels (University Park and London: Pennsylvania State University Press)

Huheey J E 1983 Inorganic chemistry--Principles of structure and reactility (New York: Harper International) 3rd edn pp. 314-315

Nakamoto K 1978 Infrared and Raman spectra of inorganic and coordination compounds (New York: John Wiley and Sons) 3rd edn p. 234

Raju K S 1983a J. Mater. Sci. Lett. 2705

Raju K S 1983 b Bull. Mater. Sci. 567

Raju K S 1983c Cryst. Res. Technol. 181277

Raju K S 1985 J. Mater. Sci. 20756

Raju K S 1986 VIII International Conference on crystal growth, University of York, York, UK

Raju K S, Godehardt R, Hopfe J and Pippel E 1984 Cryst. Res. Technol. 191127

Riggs W M and Parker M J 1975 in Methods of surface analysis (ed.) (A W Czanderna) (Amsterdam: Elsevier Scientific Pub. Co.) Chap. 4 p. 108

Wagner C D, Riggs W M, Davis L E, Moulder J F and Mullenberg G E 1978 Handbook of X-ray photoelectron spectroscopy (Minnesota: Perkin Elmer Corporation)

Weast R C and Lide D R 1990 CRC Handbook of chemistry and physics (Boca Raton, Florida: CRC Press Inc.) B-109 